\title{
Changes in differential threshold in patients with glaucoma during prolonged perimetry
}

\author{
ANDERS HEIJL* AND STEPHEN M. DRANCE \\ From the Department of Ophthalmology, University of British Columbia, Vancouver, Canada
}

SUMMARY Patients with chronic open-angle glaucoma and others suspected of having glaucoma were subjected to continuous contrast threshold measurement by automatic and manual perimetric techniques. The results show that an increase of contrast threshold over time is a frequent finding in glaucoma patients and that this increment can be large, especially at points close to existing field defects. it is reproducible and independent of background illumination and can be demonstrated with both automatic and manual testing, but it is larger in the automatic than in the manual mode of testing. The results may have some important implications for visual field testing in glaucoma.

In patients with disease of the optic pathways posterior to the lamina cribrosa a marked increase in the differential threshold has been reported during 'flashing repeat static testing.' The reduction in sensitivity which accompanies the raised threshold was termed 'visual saturation or visual fatigue-like effect.' 12 The phenomenon was described with high background luminosities of $31 \mathrm{~cd} / \mathrm{m}^{2}(100 \mathrm{asb})$ and was lessened or eliminated by diminished background luminosity. The changes were reported to be consistent in patients with optic nerve disease behind the lamina cribrosa and were not present in patients suffering from glaucoma. ${ }^{34}$ Using automatic perimetry others have reported that normal subjects maintained a stable level of differential threshold during a continuous 30 -minute examination, while many glaucoma patients showed a fairly marked increment of contrast threshold during such a test. Larger changes of threshold often occurred in areas near field defects or of relative disturbance. ${ }^{56} \mathrm{~A}$ reduction of the stimulus exposure time from $0.5 \mathrm{~s}$, which was the standard in these investigations, to $0.25 \mathrm{~s}$ augmented these changes. ${ }^{6}$

The apparent discrepancies of these findings prompted us to study the time dependence of the differential threshold during automatic and manual perimetry of glaucoma patients. We also decided to

\footnotetext{
*Anders Heijl is currently at the Department of Ophthalmology at the University of Lund, Malmo, Sweden.

Correspondence to Dr S. M. Drance, Department of Ophthalmology, University of British Columbia, 2577 Willow Street, Vancouver, BC, Canada V5Z 3N9.
}

see whether a deterioration could be observed by means of manual perimetry and whether there was an association between changes in the threshold and location of the stimulated area with relation to visual field defects. The reproducibility and dependence on the background illumination were also studied, as was the correlation between automatic and manual modes of testing.

\section{Material and methods}

All automatic perimetry was performed with the Competer computerised automatic perimeter. ${ }^{7}$ Six points were tested in each patient. A short pretest of approximately 1 minute was used to establish the initial thresholds of the points to be tested. The patients were then allowed to rest with their eyes closed for 5 minutes, and after that a continuous recording of differential thresholds was carried out for approximately 30 minutes. The computer used as its starting point the threshold measurements obtained at the pretest. The differential threshold was determined through a repetitive up-and-down staircase method. ${ }^{5}$ The 30 -minute test was continued without interruption but was subsequently divided into 12 equal periods for calculation of results. During each period the stimulus was presented 10 times at each of the 6 test points and in the blind spot of the tested eye. The order in which the stimuli were presented was randomised. Any stimulus seen was always followed by a stimulus $0 \cdot 3 \log$ unit fainter, and any stimulus not seen was increased by $0 \cdot 3 \log$ unit. The reliability of fixation was ascertained by directing 
the stimuli into the blind spot. The computer also calculated the mean of the intensity levels used at each point during each of the 12 periods and printed out a fixation quotient.

The duration of the stimulus was $0 \cdot 25$ second, and a stimulus diameter 14 minutes and background illumination $1.0 \mathrm{~cd} / \mathrm{m}^{2}$ were used. In order to study the effects of the background illumination, tests were also done with $0 \cdot 1$ and $10 \mathrm{~cd} / \mathrm{m}^{2}$ backgrounds.

All manual perimetry was performed on a Tübingen perimeter, and the testing procedure described by Sunga and Enoch ${ }^{1}$ was followed as closely as possible. Prior to the test the patients kept their eyes closed for a few minutes. The stimulus was then presented every $1 / 2$ to 1 second with an exposure time of $100 \mathrm{~ms}$. It was first presented with subliminal intensity, and the intensity was rapidly increased in steps of $0 \cdot 1 \log$ unit, being exposed several times at each level until the patient indicated that the stimulus was seen. The intensity was then decreased by 0.3 to $0.5 \log$ unit and the procedure repeated. The tests were continued manually for approximately 12 minutes at each test location. The perimetrist presented the stimuli and checked fixation while the threshold values and time were recorded by an assistant. The background intensity was usually $20 \mathrm{~cd} / \mathrm{m}^{2}$, but in some patients a background of $10 \mathrm{~cd} / \mathrm{m}^{2}$ was tested as well.

The points tested were chosen so as to be in an area of relative visual field defect or a suspect area close to an existing visual field defect as plotted on the Tübingen perimeter. In many instances each point was matched by testing the corresponding mirror point in the area of the normal visual field. The patients were corrected for their refractive errors and given a full correction for near vision, both for the automatic and manual perimetry. On manual perimetry the changes of differential threshold were calculated as the differences between the mean threshold during the first minute and the last minute of the test, while in automatic perimetry the changes were calculated as the difference between the average threshold during the first period and the 12th period.

Twenty-one patients with glaucomatous visual field defects were tested. One showed unreliable fixation during the test and the results were not included. Eleven patients were tested both manually and automatically, and 10 were tested only on the automatic perimeter. Two patients with suspect glaucoma but no visual field defects were also tested but only on the automatic perimeter.

\section{Results}

Eighteen of the 20 patients had the visual field defects in the central part of the field which was the area of the continuous automatic or manual differential threshold recording. In the 11 patients who were tested manually 20 points were tested, as it is impossible by means of manual perimetry to establish thresholds on more than one point during the same sequence of time. Eleven of these points showed stable thresholds, i.e., $-0 \cdot 2$ to $+0 \cdot 1 \log$ unit over a 12-minute period, while 9 points showed the increase in the differential threshold $(+0 \cdot 3$ to $+1 \cdot 0 \log$ unit) with a mean change of $+0.54 \mathrm{log}$ unit. In the automatic mode 108 points ( 6 in each patient) in the 18 patients tested showed much greater changes in differential threshold with time. Fourteen of the 108 points tested showed very few responses by patients during the entire 12-minute period, as they happened to be situated in an unexpectedly dense part of the visual field defect. Seventeen other points showed a marked change in the threshold, and after some time of testing the maximum luminosity of the automatic perimeter was reached, so that the resulting curve relating threshold with time could no longer be followed (Fig. 1). The mean increase in threshold of those 17 points was $+0.91 \log$ unit (SD 0.39), with the maximal change being +1.7 log units. In the remaining 77 points the mean increase of the

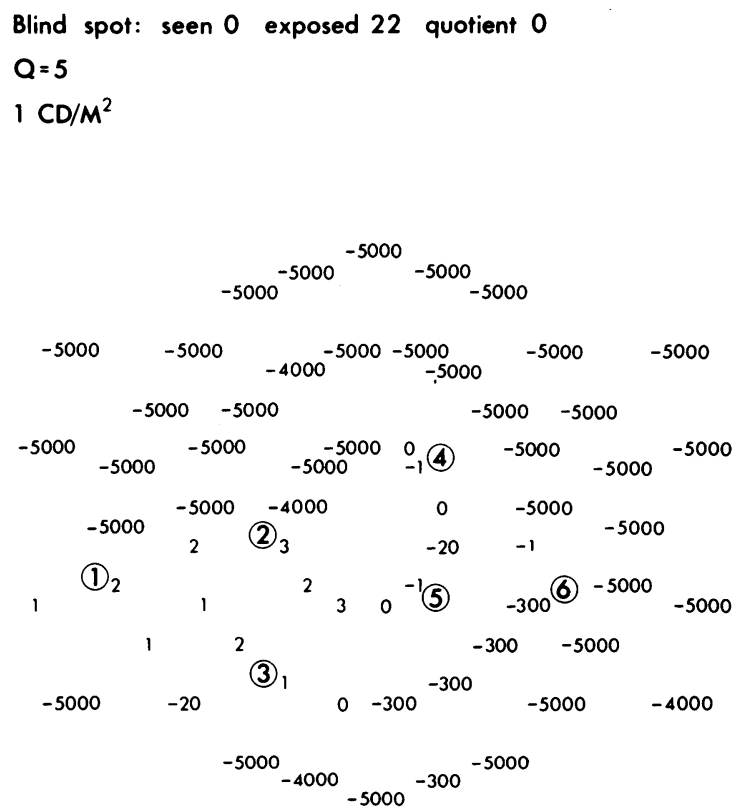

Fig. 1 Competer visual field from a right eye with chronic capsular glaucoma. The points tested are situated $5-20^{\circ}$ from fixation with three circles at 5,10 , and $15^{\circ}$ respectively. Negative numbers indicate areas with depressed sensitivity and the field thus shows a complete Bjerrum scotoma upwards and an arcuate scotoma connecting with the blind spot inferiorly. The location of the points illustrated in Fig. 2 are shown by circles. 
threshold was $+0 \cdot 27 \log$ unit (SD $0 \cdot 36$ ). Fifty points showed an increase in differential threshold greater than $0.3 \log$ unit, while only 5 points showed a decrease in threshold greater than $0.3 \log$ unit, and in only one of those was the decrease greater than 0.4 $\log$ unit.

In 4 patients who had either no field defects at all, or whose visual field disturbance was not in the central field, the mean threshold change was only $+0.01 \log$ unit.

In the 3 patients whose tests were performed at 3 levels of background luminance the results were very similar at all 3 background illuminations (Fig. 1). At

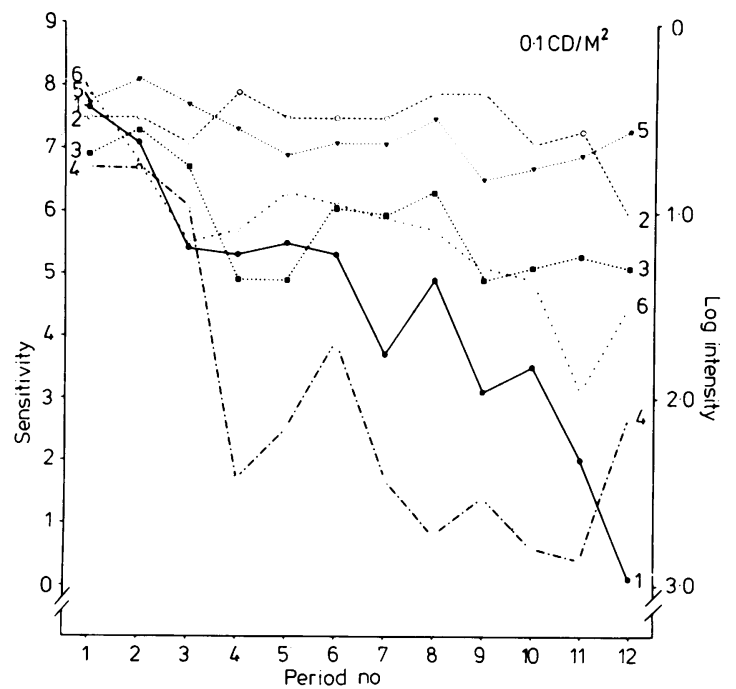

Fig. 2a

Fig. 2 Continuous automatic threshold recording in the 6 test points indicated in Fig. 1. The 30-minute test has been divided into 12 equal periods and each curve shows the sensitivity versus time in one point. The y axis shows the sensitivity levels of the Competer on the left side and the relative stimulus intensities in arbitrary units on the right side. Some points show pronounced deterioration of sensitivity with increasing test time, and this occurs at all 3 background levels $\left(0 \cdot 1,1 \cdot 0\right.$, and $\left.10 \mathrm{~cd} / \mathrm{m}^{2}\right)$. The location of points $1-6$ tested are shown in Fig. 1. The most stable points 2 and 5 are situated closest to fixation in the least disturbed inferior half of the field. the lowest background illumination fewer of the curves were truncated because of the greater margin between the initial threshold and the maximum stimulus luminosity of the computerised perimeter. Two of these patients were tested manually at 2 background luminosities, and the results were very similar.

The 6 points tested in each patient on the automatic perimeter were classified into 3 groups according to their relationship to defective areas. Classification into these 3 groups was done independently and without access to the results of the continuous threshold recordings.

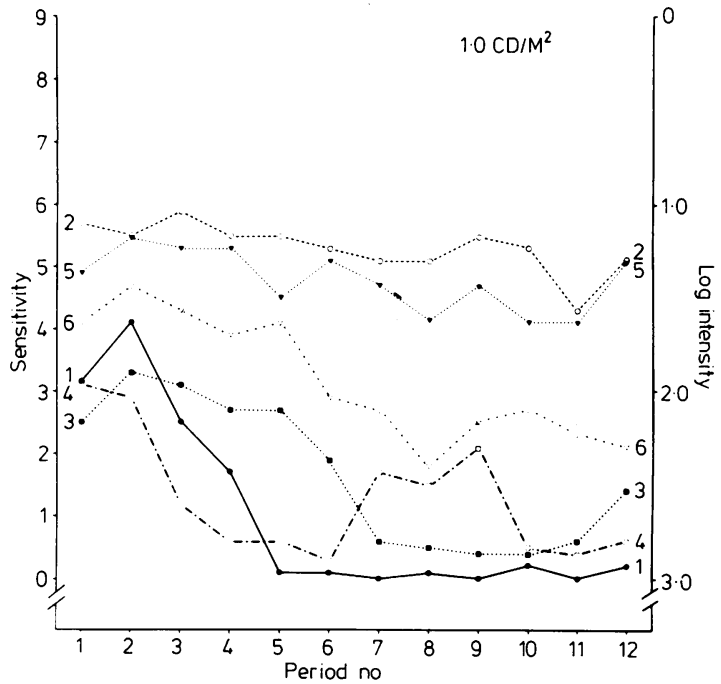

Fig. 2b

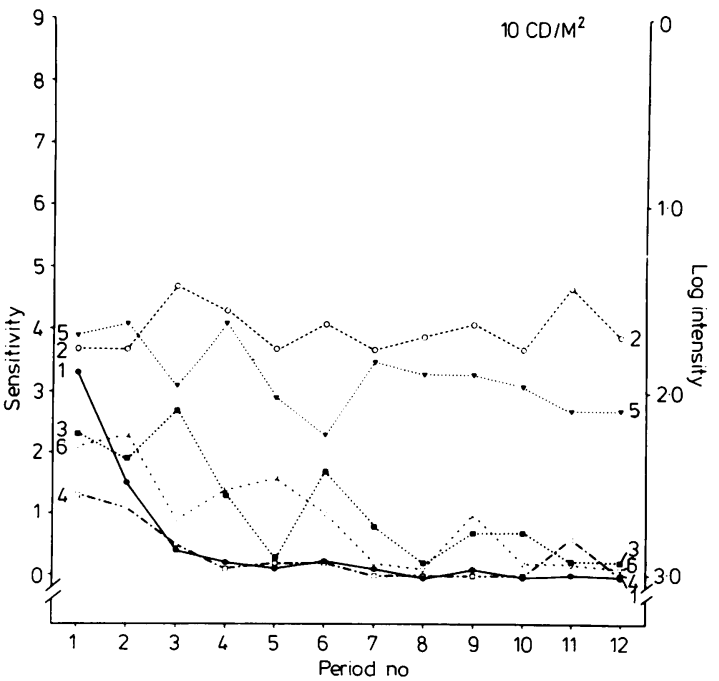

Fig. 2c 
Table 1 Mean increment of differential threshold over 30 minutes of automatic testing in relationship to proximity to existing field defects

\begin{tabular}{ll}
\hline Patient group & Mean increment in threshold in log units \\
\hline I & $+0.57($ SD 0.47) \\
II & $+0 \cdot 44($ SD 0.47) \\
III & $+0 \cdot 10($ SD 0.27$)$ \\
\hline
\end{tabular}

Group I: Test points from defective parts of the visual field.

Group II: Test points close to existing field defects.

Group III: Test points in normal parts of the visual field.

$\left.\begin{array}{l}\text { I }>\text { II } ; p<0.05 \\ \text { II }>\text { III } ; p<0.05\end{array}\right\}$ one-sided sign test.

I $>$ III $; \mathrm{p}<0.01$

Group 1 included points from defective parts of the visual field. Group 2 consisted of those points outside an existing field defect but so situated that if any progression in the visual field defect were to occur it might involve the point tested. Group 3 consisted of points in the normal parts of the visual field well removed from existing visual field defects as judged from previous manual kinetic and static and automatic fields.

In 14 points which happened to be in more grossly defective areas than suspected from manual perimetry very few responses were elicited, and they could therefore not be used in the analysis. The mean increase in differential threshold of the points in each of the above 3 groups is presented in Table 1 . The results show that, during a 30-minute automatic perimetric testing session, points tested in a relative visual field defect or in areas of the visual field adjacent to a visual field defect showed a greater increase in threshold than points situated in normal areas of the visual field away from existing defects $(p<0.01$ and $p<0.05$ respectively-one-sided sign test). The means given for groups 1 and 2 are probably an underestimate of the actual change, as some of the threshold changes reach the maximum stimulus intensity of the automatic perimeter and the curves relating threshold increment and time were therefore truncated.

There was a statistically significant correlation between the results obtained on manual and automatic testing (Spearman's rank correlation coefficient $=0.49 ; \mathrm{p}<0.05$-one-sided test). The changes in threshold on manual testing were usually a good deal smaller (Fig. 3). In those points tested with both methods the mean increment of the threshold on automatic testing was $+0.67 \mathrm{log}$ unit, whereas on manual testing it was $+0 \cdot 19 \log$ unit. The greater change shown on automatic perimetry was present even when the increment was calculated at the end of 12 minutes of the 30-minute automatic testing in order to correspond to the average duration of the

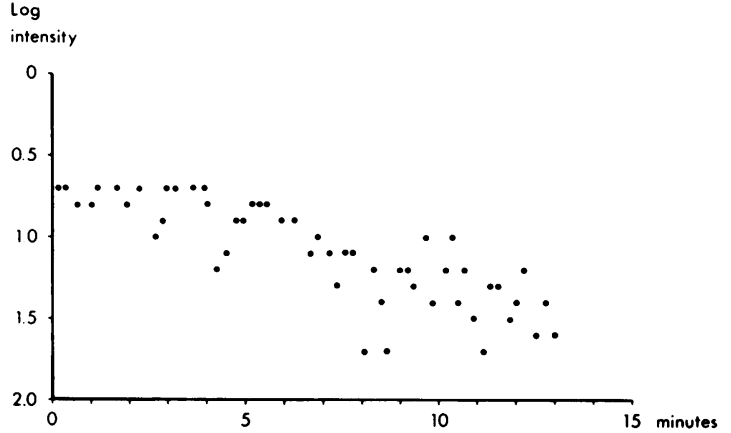

Fig. 3a

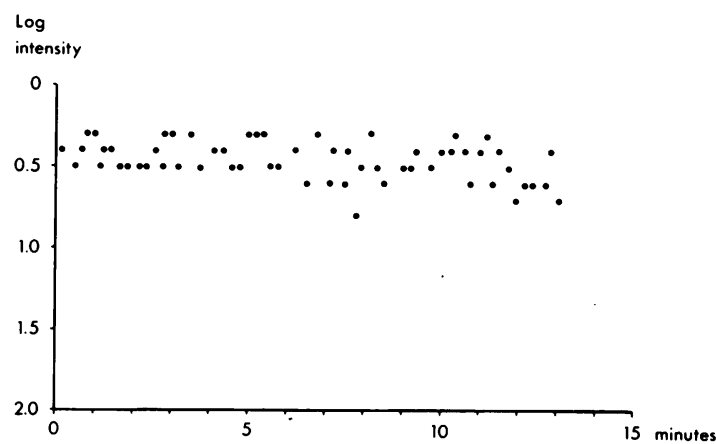

Fig. 3b

Fig. 3 Result of continuous manual threshold recording in an eye with chronic simple glaucoma. Log stimulus intensity on the y axis. Two points have been tested, one in a slightly disturbed part of the field (Fig. 3a) and one in the corresponding mirror point in a normal part of the field (Fig. 3b). The test point in the pathological part of the field shows decreased sensitivity with time, while the point in the normal part of the field remains more stable.

manual tests. As one might have expected the correlation between the 12-minute automatic results and the manual results was better than between the 30minute automatic and the manual results (Spearman's rank correlation coefficient $=0.77 ; \mathrm{p}<0.01$ - onesided test).

No difference was found between the results obtained from patients with chronic open-angle glaucoma and those with low-tension glaucoma. There was also no difference between patients who had recently shown progression of their visual field defects and those whose visual fields were stable.

\section{Discussion}

The study suggests that

(1) an increment of the differential threshold during prolonged continuous threshold recording is common in patients with glaucoma; (2) this increment in 
threshold was demonstrated both in automatic and manual perimetry; (3) the increment is larger in the automatic than manual mode of perimetry, which is in keeping with the greater changes in the differential threshold normally seen by means of automatic perimetry as compared with its manual counterpart; (4) areas in a relative scotomata or immediately adjacent to it show a more pronounced deterioration of threshold than normal points well away from field defects; (5) the increases of threshold occur at different background luminosities, both in automatic and manual perimetry; and (6) those points showing an increase of threshold manifested them both at automatic and manual testing, whereas those points being stable were usually stable in both modes of testing.

There was a definite deterioration of the differential threshold with time during continuous threshold determinations. In accordance with 2 previous studies $^{56}$ we found this phenomenon common in patients with glaucoma. The present study further shows that it can be elicited on manual perimetry and is independent of background luminosity. These results are different from those of Enoch and colleagues, ${ }^{34}$ but he showed increases of threshold of 1 or $2 \log$ units over a 5-minute period in patients with disease behind the optic disc, ${ }^{12}$ while in our studies the increases were often smaller and appeared later during the test. Since the changes are independent of the background luminosity it must be questioned whether these are really the same saturation-like phenomena which Enoch and colleagues described. We used the optimum eccentric lens correction giving the minimum threshold ${ }^{8}$ in only a few cases, but since these showed the same type of the response as the rest of the patients we have no reason to believe that this could play any major role in our results.

Since the threshold changes in patients with glaucoma may still be quite large, especially near to existing field defects, they may have important implications. Firstly, one cannot assume an increase in the accuracy of measuring the differential threshold if procedures like averaging of many responses are used and a continuous mode of testing is utilised. Secondly, the length of a test in a glaucoma patient should preferably be standardised if one wants to use the information to determine whether a glaucomatous field defect has progressed or not. Thirdly, the present results indicate that a moderate decrease in sensitivity at repeated static testing cannot be taken as a sign pointing only to a nonglaucomatous lesion. Further, the increment of differential threshold during prolonged testing might be a sign of impending damage, because it is much more common in areas where one might expect progression of a field defect to occur as compared with those areas well away from a scotoma. It might therefore provide information about early disturbance and be a possible perimetric provocative test, indicating the possibility of subsequent visual field damage. But to test this will require prospective studies.

This work was supported in part by the E. A. Baker Foundation, Medical Research Council of Canada, MT 1578, Swedish Medical Research Council grant B81-17F-5900-01, and Alcon Pharmaceuticals Ltd.

\section{References}

1 Sunga RN, Enoch JM. Further perimetric analysis of patients with lesions of the visual pathways. Am J Ophthalmol 1970; 70: 403-22.

2 Enoch JM, Berger R, Birns R. A static perimetric technique believed to test receptive field properties: extension and verification of the analysis. Doc Ophthalmol 1970; 29: 127-53.

3 Enoch JM. Quantitative layer-by-layer perimetry. Invest Ophthalmol Visual Sci 1978; 17: 199-257.

4 Enoch JM, Campos EC. Analysis of patients with open-angle glaucoma using perimetric techniques reflecting receptive fieldlike properties. Doc Ophthalmol Proc Ser 1979; 19: 137-49.

5 Heijl A. Time changes of contrast thresholds during automatic perimetry. Acta Ophthalmol (Kbh) 1977; 55: 696-708.

6 Holmin C, Krakau CET. Variability of glaucomatous visual field defects in computerized perimetry. Albrecht von Graefes Arch Klin Ophthalmol 1979; 210: 235-50.

7 Heijl A, Krakau CET. An automatic perimeter for glaucoma visual field screening and control. Albrecht von Graefes Arch Klin Ophthalmol 1975; 197: 13-23.

8 Fankhauser F, Enoch JM. The effects of blur upon perimetric thresholds. Arch Ophthalmol 1962; 68: 240-51. 